\title{
A compact electron cyclotron resonance proton source for the Paul Scherrer Institute's proton accelerator facility
}

\author{
C. Baumgarten, ${ }^{\text {a) }}$ A. Barchetti, H. Einenkel, D. Goetz, and P. A. Schmelzbach \\ Paul Scherrer Institute, Switzerland
}

(Received 14 February 2011; accepted 21 April 2011; published online 25 May 2011)

A compact electron cyclotron resonance proton source has been developed and installed recently at the Paul Scherrer Institute's high intensity proton accelerator. Operation at the ion source test stand and the accelerator demonstrates a high reliability and stability of the new source. When operated at a $10-12 \mathrm{~mA}$ net proton current the lifetime of the source exceeds $2000 \mathrm{~h}$. The essential development steps towards the observed performance are described. () 2011 American Institute of Physics. [doi:10.1063/1.3590777]

\section{INTRODUCTION}

The high intensity proton accelerator facility at the Paul Scherrer Institute (PSI)(Refs. 1 and 2) consists of a $810 \mathrm{kV}$ Cockcroft-Walton (CW) pre-accelerator, ${ }^{3,4}$ a $72 \mathrm{MeV}$ injector cyclotron (INJECTOR II), and the $590 \mathrm{MeV}$ Ring cyclotron. The extraction system of the source located in the high voltage terminal of the $\mathrm{CW}$ runs at $60 \mathrm{kV}$. The beam extracted from the source is focused, adjusted and collimated with two solenoids, four steering magnets and two watercooled diaphragms before entering the accelerating tube of the CW. A fast kicker magnet enables to pulse or shut off the beam. The beam from the pre-accelerator is transported by the $870 \mathrm{keV}$-beamline through a system of two bunchers, a $50 \mathrm{MHz}$-buncher and a third harmonic $150 \mathrm{MHz}$-buncher and then injected into the INJECTOR II cyclotron. This accelerator is a 4-separate sector cyclotron running at $50 \mathrm{MHz}$ and is optimized to operate in a space charge dominated regime without flattop cavity.

A multicusp ion source driven by tungsten filaments has been used for many years. ${ }^{5-7}$ This source had disadvantages, the main being the short maintenance intervals of two weeks and the sensitivity to changes of the operating conditions. Besides that, the proton fraction is relatively low and the net proton current is limited to about $11 \mathrm{~mA}$. To satisfy the call of the users of the facility for three weeks uninterrupted running periods, the source had to be replaced by a new device with service intervals of three weeks or a multiple thereof.

Very good performances have been demonstrated with proton sources based on the ECR technique. ${ }^{8-10}$ These sources are however not suitable for installation in the limited space available in the CW-terminal. On the other hand, the proton currents achieved by these devices largely exceed our needs. The compact source developed by Cui et al. ${ }^{11}$ triggered our decision to design and test a similar source for the PSI accelerator. The limiting factors for the lifetime of this type of source are plasma abrasion and damage of the plasma chamber bottom by backstreaming electrons. Therefore, special attention was given to a design allowing for a fast replacement of this component.

\footnotetext{
a)Electronic mail: christian.baumgarten@psi.ch.
}

\section{THE SETUP OF THE ECR SOURCE}

The design of the source is kept as simple as possible. It consists of a $2.45 \mathrm{GHz}$-microwave system, an assembly of SmCo permanent magnets with a trim coil providing the resonant magnetic field of $87.5 \mathrm{mT}$ inside a $50 \mathrm{~mm}$ diameter, and $38.5 \mathrm{~mm}$ long plasma chamber.

The length of the chamber has been experimentally optimized and corresponds to $\approx \lambda / 4$ in a WR284 waveguide. Since the magnetic field increases with the distance from the axis, the diameter of the resonance zone is radially limited, but depends on the current in the trim coil and the temperature of the permanent magnets. The radius of the chamber is about twice the maximal radial extension of the ECR-resonance zone.

The setup of the proton source is depicted in Fig. 1.

A permanent magnet system with three stacks of SmComagnets ${ }^{12}$ is mounted in a rectangular geometry around the waveguide surrounding the plasma chamber. It is designed to generate a two-bump, slowly varying axial magnetic field of $\sim 87.5 \mathrm{mT} .{ }^{13}$ The magnet stacks are spaced by a trim coil that allows to fine-tune the ECR-resonance condition and by a water cooled copper plate that keeps the magnet system at constant temperature. A stainless steel frame hooks up the collar of the plasma chamber to a water cooled copper base plate. Compressed air is blown into the last waveguide section in order to cool the plasma chamber. The whole assembly is $140 \mathrm{~mm}$ in diameter and $80 \mathrm{~mm}$ long. The shape of the magnetic field along the axis is shown on Fig. 2. The plasma chamber is sealed with a high temperature O-ring ${ }^{14}$ towards the base plate. The temperature range of the O-ring limits the maximal microwave power to $\sim 700 \mathrm{~W}$, but under typical conditions the maximal proton fraction is already obtained at lower power. The magnet stack is separated from the extraction electrodes by an iron plate (ST 37) with a tungsten insert containing a tapered extraction aperture of $4.5 \mathrm{~mm}$ diameter. The iron plate works as a magnetic mirror - which is essential for the configuration of the magnetic field - and as a shield towards the high voltage region.

The microwave (MW) power system is similar to those used in other sources of this type: ${ }^{15}$ A $1.2 \mathrm{~kW}$ generator ${ }^{16}$ produces $T E_{10}$-mode waves in a WR 340 waveguide, 


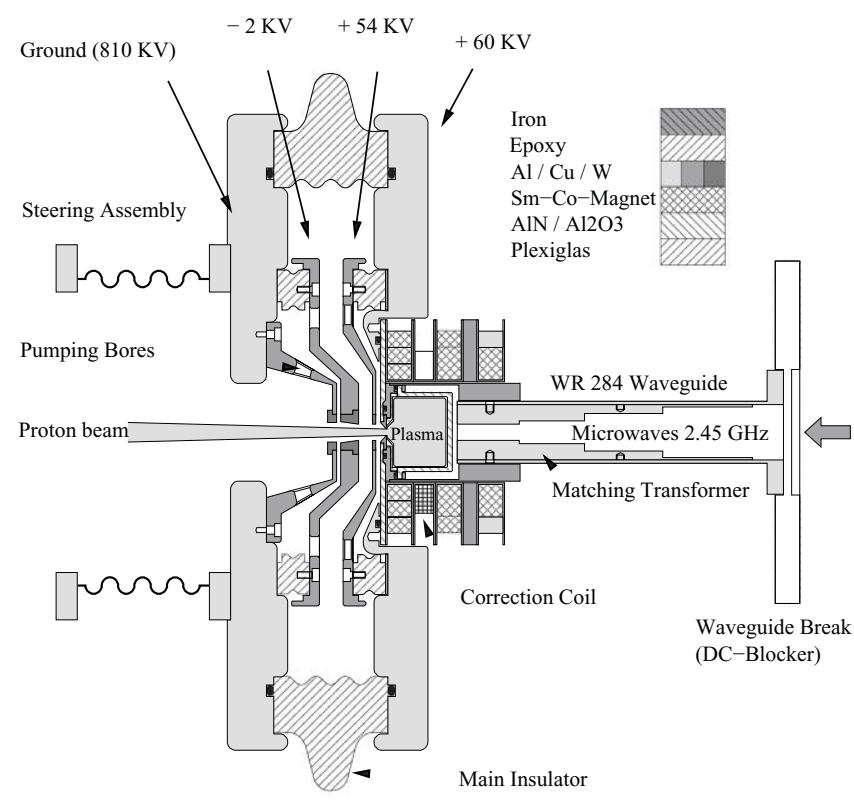

FIG. 1. Schematic of the setup of the new compact ECR proton source. Distances and apertures of the extraction electrodes are identical to the setup of the formerly used multicusp ion source.

followed by circulator/dummy load assembly to absorb the reflected power. A 4-stub automatic tuner minimizes the reflected power. ${ }^{17}$ It is followed by a $90^{\circ}$-bend and a WR340to-WR284-transition to reduce the size of the waveguide. The following waveguide break made of $5 \mathrm{~mm}$ thick plexiglas acts as a dc-blocker and, thus, allows to keep the microwave generator and tuner on ground potential. The last part of the waveguide is slipped into a copper case surrounding the plasma chamber. This part contains a 4-step rectangular-to-ridged binomial matching transformer which improves the matching to the plasma impedance and concentrates the microwave power on axis. ${ }^{18}$ The reflected power can be monitored by a detector diode at the dummy load. Both sources, the assembly and the extraction system are hooked with a two-axis steering as-

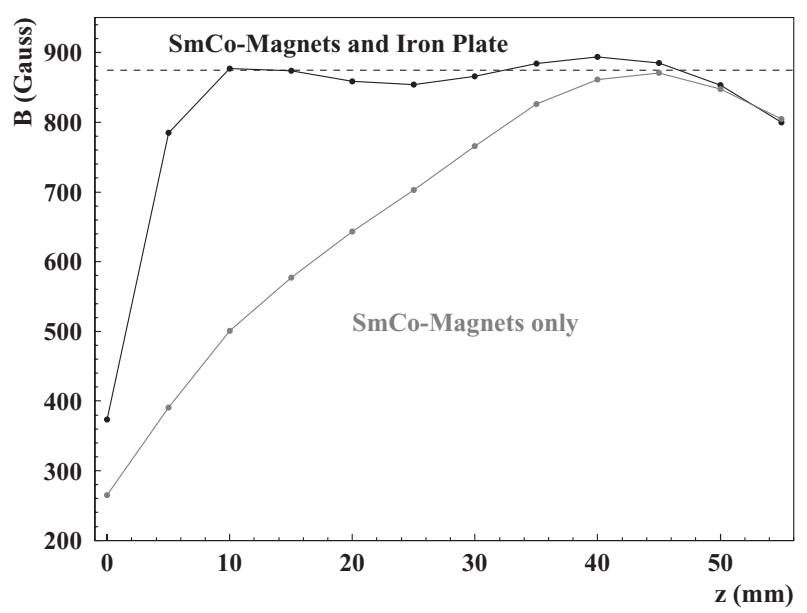

FIG. 2. Black: Magnetic field on axis of the plasma chamber. Gray: Field without the iron extraction plate. The dashed line indicates the ECR resonance at $875 \mathrm{G}$. The plasma electrode is located at $z=0$ and the bottom of the plasma chamber is at about $z=38.5 \mathrm{~mm}$. sembly to a vacuum chamber equipped with a 20001/s turbomolecular pump. The steering assembly allows to align the beam with the direction of the beam transport system. A gas flow controller provides $\mathrm{H}_{2}$ gas, which is guided through a stainless steel capillary soft-soldered to the copper base plate of the plasma chamber. The gas feed line is equipped with a controllable water leak composed of a water reservoir and a remote controlled needle valve.

\section{OPTIMIZATION PHASE AND THE RESULTS OF THE TEST STAND OPERATION}

\section{A. Plasma chamber}

The optimization phase of the new source was accompanied by intense tests at the ion source test stand (ISTS). Various design details were reviewed and had to be modified as reported. ${ }^{19}$ One major problem was the choice of the plasma chamber material. Quartz was used first as it is known to have a low hydrogen recombination coefficient and low thermal expansion. The quartz chamber was protected against backstreaming electrons by a $2 \mathrm{~mm}$ thick boron nitride (BN) disc attached to the chamber bottom. Both materials turned out to be a problematic choice. The lifetime of the plasma chamber was limited due to plasma etching of the walls. Second, high voltage breakdowns in the extraction system developed with time to a serious problem as they eventually occurred at an unacceptable high frequency of up to $0.1 \mathrm{~min}^{-1}$. The breakdowns were apparently caused by the deposition of BN dust and the glassy black grains on the extraction electrodes. Similar deposits have also been found in other sources. ${ }^{20}$ The high sparking rate in turn caused metal (copper) evaporation in the extraction system which entered the plasma chamber and condensed at the BN disc as a colored layer. This caused a reflection of the microwave power so that the plasma stopped igniting typically after some days of operation.

At first glance an alternative material for the plasma chamber might be pure alumina $\left(99 \% \mathrm{Al}_{2} \mathrm{O}_{3}\right)$. With an alumina chamber the average time between high voltage breakdowns reached several hours. Furthermore, the efficiency of the source improved significantly since alumina is known as an electron donor. ${ }^{21}$ Unfortunately, alumina has a higher thermal expansion coefficient which makes its use critical under local thermal stress. In fact, this approach was not successful since several plasma chambers cracked after some hours of operation. The cracks always started at the center of the bottom wall indicating that the intense local heating by the backstreaming electrons was responsible for the failure. Finally an aluminum nitride (AlN) plasma chamber has been chosen. The ratio of beam current to microwave power is slightly below that of the alumina chamber, but the thermal conductivity of AlN is about 6-8 times larger and the thermal expansion is below that of alumina. Several chambers were tested during runs of up to $1400 \mathrm{~h}$. No damages were observed at the walls and also the backstreaming electrons did not cause noticeable damage.

Water vapor is known to reduce wall recombination in HF-driven hydrogen discharges. According to Spence and Lykke a $\mathrm{H}_{2} \mathrm{O}$-admixture of $1 \%-2 \%$ to the hydrogen gas can 


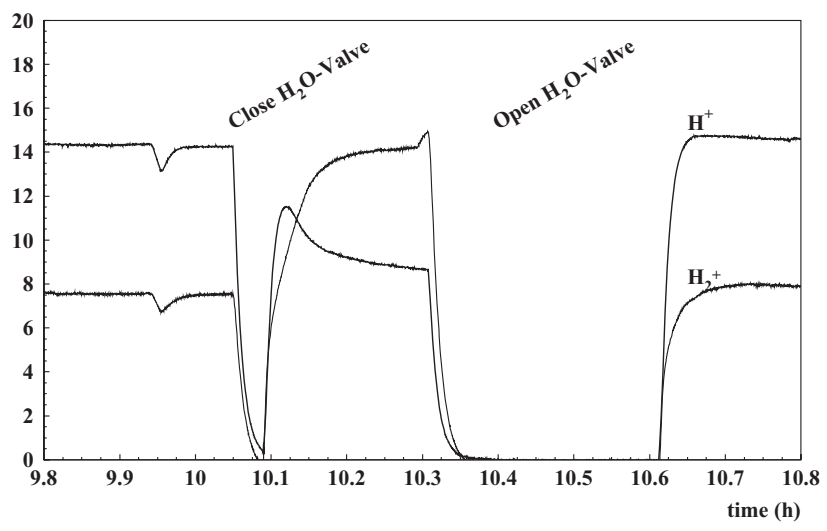

FIG. 3. Ion beam fractions with and without $\mathrm{H}_{2} \mathrm{O}$-admixture. The plot shows the temperature increase of the cooling water of the beam dump at the end of the beamline $\left(\mathrm{H}^{+}\right)$and of the collimator BV4 which blocks the majority of the $\mathrm{H}_{2}^{+}$-ions (and the beam tails of the $\mathrm{H}^{+}$-beam). The reaction of the plasma to the $\mathrm{H}_{2} \mathrm{O}$-admixture is fast. Measurements of the mass spectra as shown in Fig. 6 verified the effect.

help to increase the proton fraction of the beam from an ECR source. ${ }^{22}$ Their results could be reproduced after the installation of a water reservoir connected by a needle valve to the gas feed line. Figure 3 shows the behavior of the beam when this needle valve is closed and opened again. There are indications that the $\mathrm{H}_{2} \mathrm{O}$-admixture helps to remove surface contaminations and increases the lifetime of the source as well.

The extraction electrode system is the same as for the multicusp source. It consists of the extraction plate on a potential of $60 \mathrm{kV}$ relative to the $\mathrm{CW}$, an intermediate electrode adjustable within $50-59 \mathrm{kV}$ which is used to optimize the beam focus, the electron trap at a negative potential of typically from -1 to $-2 \mathrm{kV}$, and finally the ground electrode.

\section{B. Vacuum system}

The thermal load of the bottom of the plasma chamber by the plasma and by backstreaming electrons is the main issue in this type of source. Therefore, other components of the system also have to be optimized with respect to this problem. The backstreaming electrons are produced by proton impact ionization of neutrals in the extraction system. The electric field accelerates the electrons back towards the plasma chamber where they are focused on axis by the magnetic field resulting in a high power density on axis. One way to reduce the electron current is to lower the gas pressure of neutrals in the extraction system. Therefore, additional pumping holes were machined in the extraction electrodes to reduce the rest gas pressure between the electrodes, for example from $2.3 \times 10^{-3}$ mbar to $3 \times 10^{-4}$ mbar at a gas flow of $5 \mathrm{sccm}$. Figure 4 compares calculated pressures and resulting electron currents on axis for the cases with and without these pumping bores and an extracted current of $26 \mathrm{~mA}$ according to

$$
\frac{I_{e}(z)}{I_{p}}=\frac{1}{k_{b} T} \int_{z^{\prime}=0}^{z} \sigma_{i}\left(E\left(z^{\prime}\right)\right) p\left(z^{\prime}\right) d z^{\prime},
$$

where $I_{e}\left(I_{p}\right)$ are the electron (proton) current, $\sigma_{i}(E)$ is the proton impact ionization cross section, ${ }^{23} p$ is the neutral gas pressure, $k_{b}$ is the Boltzmann constant, and $T$ is the gas tem-

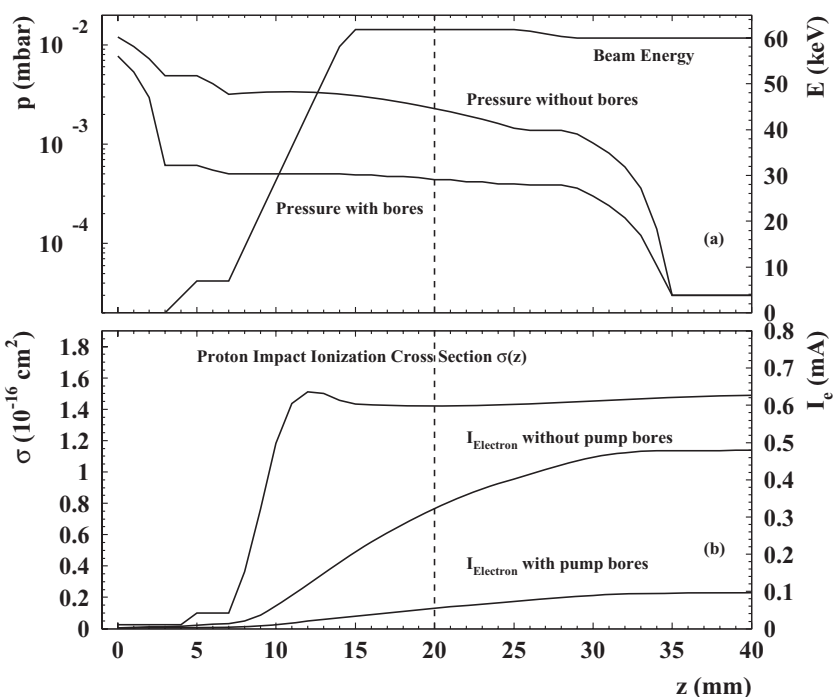

FIG. 4. (a): Calculated pressure and beam energy along the axis starting at the extraction $(z=0)$. (b): Calculated proton impact ionization cross section of $\mathrm{H}_{2}$ and calculated current of the backstreaming electrons produced by proton impact ionization of hydrogen gas in the extraction system for a total beam current of $26 \mathrm{~mA}$. The dashed line indicates the position $z_{m}$ of maximal beam energy. Beyond that point $\left(z>z_{m}\right)$ electrons are pushed outward by the negative potential of the electron trap.

perature. One calculates up to $0.3 \mathrm{~mA}$ of electron current corresponding to a heat load of about $16 \mathrm{~W}$. For an estimated impact area of $10^{-3} \mathrm{~cm}^{2}$ the power density reaches $16 \mathrm{~kW} / \mathrm{cm}^{2}$. The improved vacuum conditions reduce the power of the backstreaming electrons by a factor of five.

But it should also be pointed out that the $\mathrm{H}_{2}$ flow giving optimal results increased by this measure from $2.5 \mathrm{sccm}$ to about $5 \mathrm{sccm}$. The main reason is apparently the required supply of electrons from the ionization of rest gas molecules for space charge compensation.

\section{Beam emittance and proton fraction}

The setup of the ISTS is analogues to the CW-setup. The ECR source and the extraction system are surrounded by the high voltage hut. A girder of $\sim 3.5 \mathrm{~m}$ length allows to mount various low energy beam transport (LEBT) elements and diagnostic chambers. A system of two moveable slits has been used for emittance measurements. A water cooled Faraday cup at the end of the LEBT is used as a beam dump as shown in Fig. 5. A dipole magnet in front of the double slit system was optionally used to measure the contributions of $\mathrm{H}^{+}, \mathrm{H}_{2}^{+}$, and $\mathrm{H}_{3}^{+}$ions to the beam current as shown in Fig. 6, or to measure the phase space ellipses of all ionic beam components simultaneously. This is possible since the phase space ellipses of the different beam components are quite narrow in $x^{\prime}$ and are well separated by the dipole magnet as shown in Fig. 7. The slits are about $50 \mathrm{~mm}$ long and $0.2 \mathrm{~mm}$ wide. The proton fraction is typically above $90 \%$ for a new plasma chamber and degrades with time to values of $70 \%-80 \%$. The admixture of water vapor suppresses further degradation.

For a measurement of the horizontal (vertical) emittance, both slit drives were mounted horizontally (vertically). 


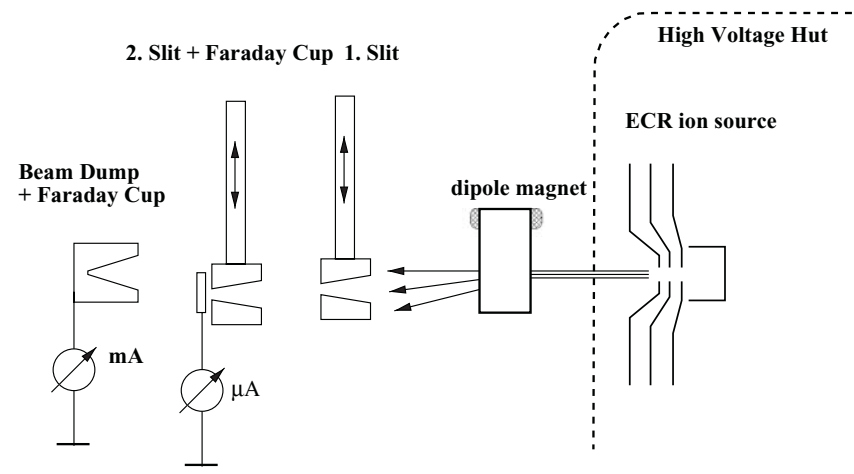

FIG. 5. Setup of the ISTS with double slit system and dipole magnet for emittance measurements and measurements of the mass spectrum.

Ion tracking computations have been performed in order to improve the understanding of the phase space occupied by the beam. For this purpose the electrical potential in the extraction region has been computed by the electrostatic solver RELAX3D (Ref. 24) (see Fig. 8). The influence of the plasma potential has been modeled by overlaying a second potential with all electrodes grounded except one virtual electrode representing the plasma potential.

The form of the plasma meniscus (zero-potential surface) can then be adjusted by using different weight factors of the electrode field and the virtual plasma potential. Tracks are started at the plasma meniscus with a direction perpendicular to the plasma meniscus. A starting energy of $10 \mathrm{eV}$ gave good agreement with the measurements and can be interpreted by the plasma potential, and should not be confused with the ion thermal energy which should be in the order of $0.1 \mathrm{eV}$. The resulting ion tracks are then used to compute the beam size and the space charge beam potential. The space charge potential is added to the electrode potential and the ion tracks have been recalculated. After $<10$ iterations the beam size converges.

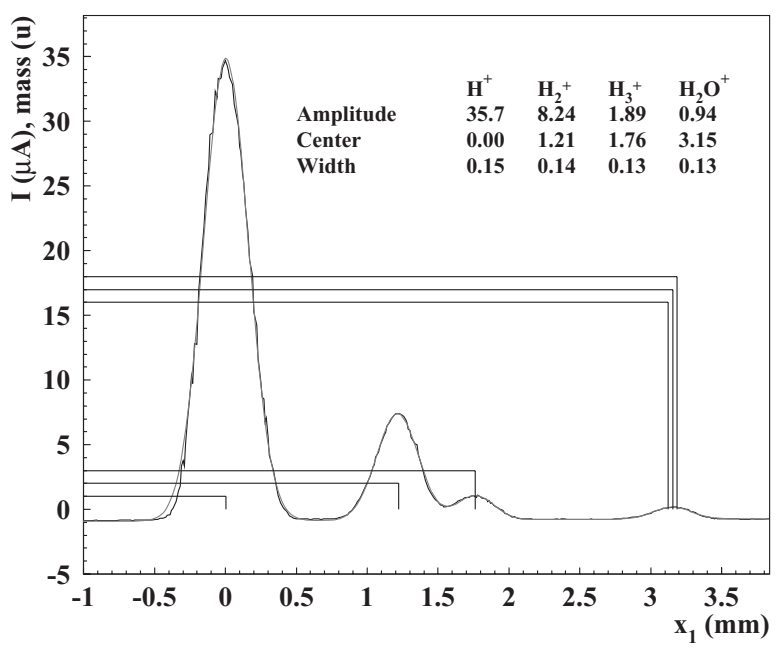

FIG. 6. Result of a mass spectrum measurement at the ISTS using the setup shown in Fig. 5. $x_{1}$ is the position of the second slit. The first slit was kept in fixed position. The mass peaks of $\mathrm{H}^{+}, \mathrm{H}_{2}^{+}$, and $\mathrm{H}_{3}^{+}$are clearly resolved. The 4th peak is the sum of $\mathrm{O}^{+}, \mathrm{HO}^{+}$and $\mathrm{H}_{2} \mathrm{O}^{+}$and indicates that the controlled water leak is in operation. The proton fraction is here approximately $76 \%$. The water vapor admixture is about $1 \%-2 \%$.

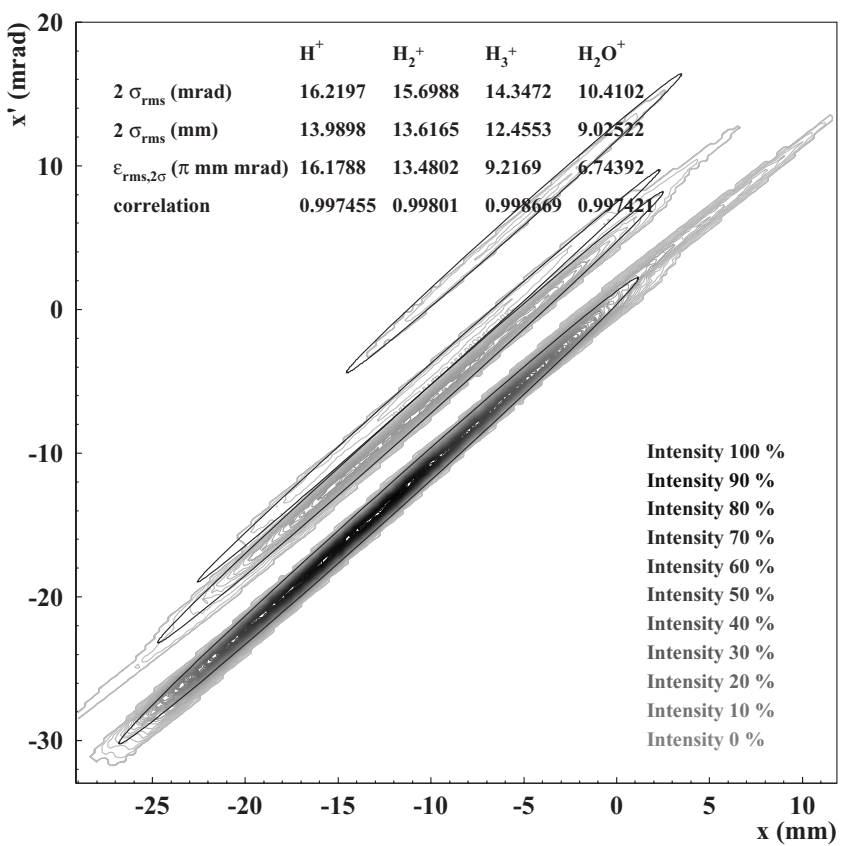

FIG. 7. Contour plot of an emittance measurement at the ISTS using the setup shown in Fig. 5. $x$ refers to the position of the first slit and $x^{\prime}$ is the difference of both slit positions devided by their distance. The dipole magnet was excited during this measurement. The phase space ellipses have been evaluated separately for the different masses and the results are drawn as black ellipses on top of the contours.
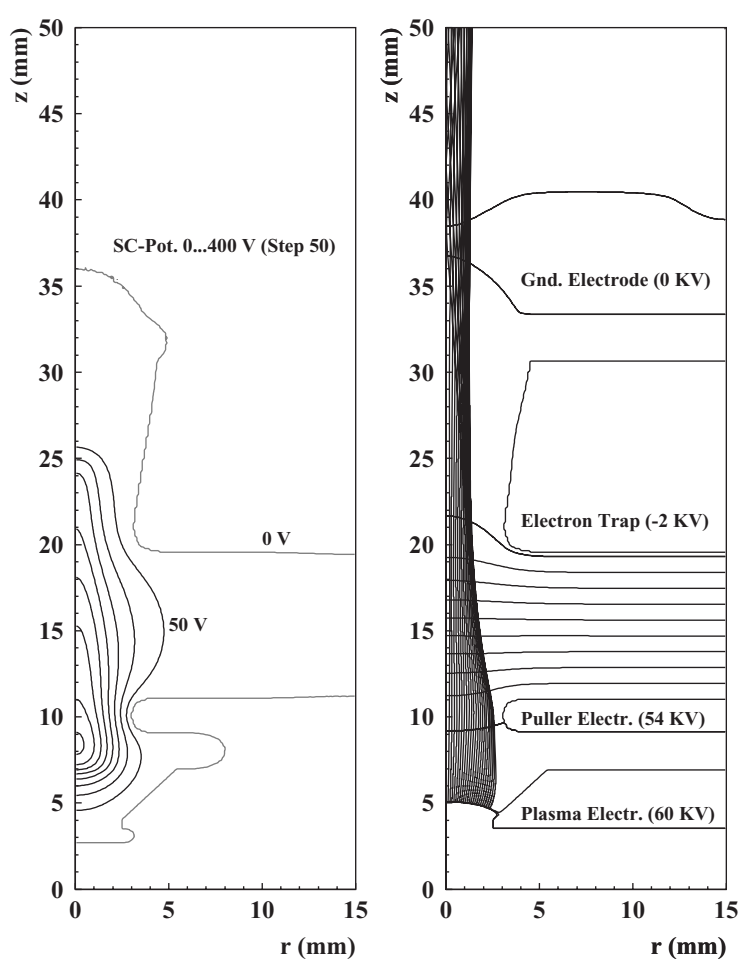

FIG. 8. Left: Space charge potential of the extracted beam current in the extraction system. Right: Electrostatic potential and ion tracks in the extraction system. Tracking starts on the zero-potential-surface called the plasma meniscus. Best agreement with the measured emittance ellipse was found for a slightly convex plasma meniscus. 


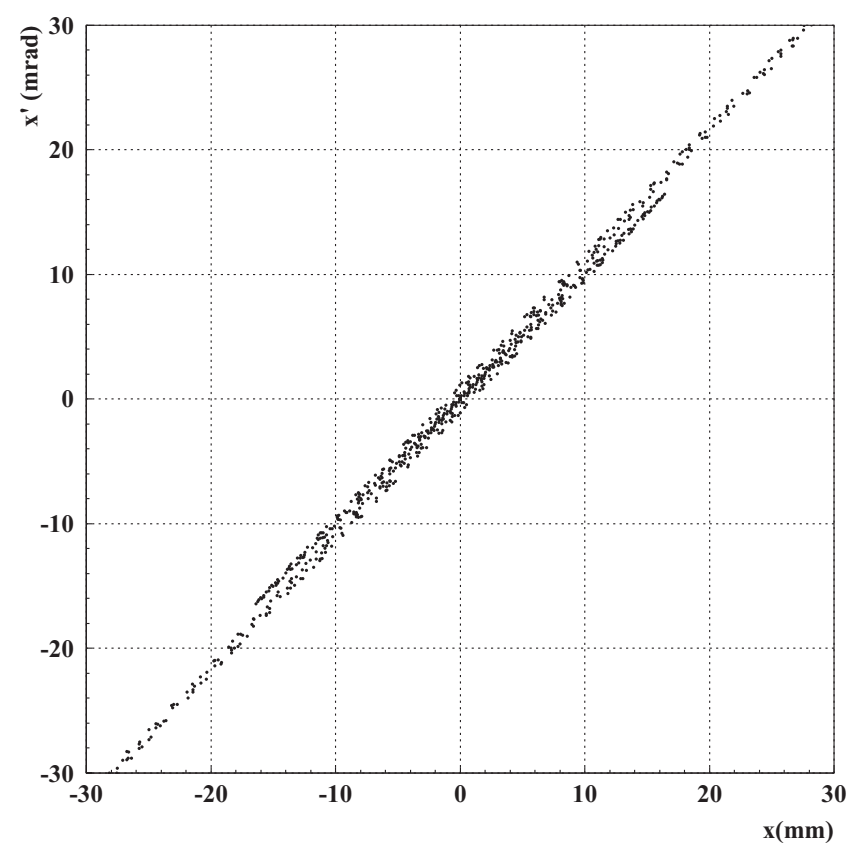

FIG. 9. Phase space plot of ions tracked from the source to the double slit system including space charge (see Fig. 8). The form and size of the phase space agrees well with the measurement (see Fig. 7).

The resulting phase space plot is in good agreement with the measurement and is shown in Fig. 9.

The horizontal and vertical $2 \sigma \mathrm{rms}$ emittances are both about $16 \pi \mathrm{mm} \mathrm{mrad}$ at a total beam current of $24 \mathrm{~mA}$ using optimal settings of the source and electrode voltages as in Fig. 8. Since the phase space indicates a very strong correlation between $x$ and $x^{\prime}$, it is possible to measure the beam shape by mounting the first slit drive in the horizontal direction and the second in the vertical direction. The measurement showed that the beam profile is nearly "round," even though the permanent magnets have a rectangular configuration, i.e., no exact rotational symmetries.

Before installation of the source in the $\mathrm{CW}$ preaccelerator, a copy of the LEBT beamline was setup at the ISTS, i.e., two solenoids, two collimators, and four steering magnets as shown in Fig. 10. After optimization of the gas flow and solenoid settings it was verified that this setup allows to transmit sufficient current $(10-12 \mathrm{~mA}$ at about $20 \mathrm{~mA}$ of total extracted current) for the proton machine.

\section{OPERATION EXPERIENCE IN THE ACCELERATOR}

After installation of the new ECR source in the shutdown 2009/2010, the beam could be transported to INJECTOR II with a few minor adjustments of the quadrupoles in the $870 \mathrm{keV}$-beamline and a beam current of $2.5 \mathrm{~mA}$ could easily be extracted from INJECTOR II. At present a maximum current of $2.2 \mathrm{~mA}$ for routine production ( $2.3 \mathrm{~mA}$ for test purposes) is allowed in the Ring cyclotron.

It was expected that the new ECR source with standard magnetron would cause a relatively high $50 \mathrm{~Hz}$ beam ripple, even with the recently improved stabilization of the $\mathrm{CW}$ power supply. At the ISTS it has been observed that the total beam current had a $50 \mathrm{~Hz}$ peak-to-peak noise of up to $2 \%$, depending on microwave power, gas flow, and tuning of the magnetic field. This noise is related to the $4.4 \mathrm{~V}$ ac heating voltage of the magnetron filament, which adds up to the anode voltage of $4-4.5 \mathrm{kV}$. This $500 \mathrm{~V}$-range of anode voltage controls the microwave output power from 0 to $1200 \mathrm{~W}$. A $50 \mathrm{~Hz}$-ripple of about $1 \%$ of the full voltage range corresponds to a power ripple of $12 \mathrm{~W}$ or about $2 \%$ at a power level of $600 \mathrm{~W}$. In combination with the non-zero internal impedance of the $\mathrm{CW}$ power supply, the intensity ripple transforms into a voltage ripple of the $\mathrm{CW}$ voltage, i.e., an energy ripple of the accelerated beam. The energy ripple then

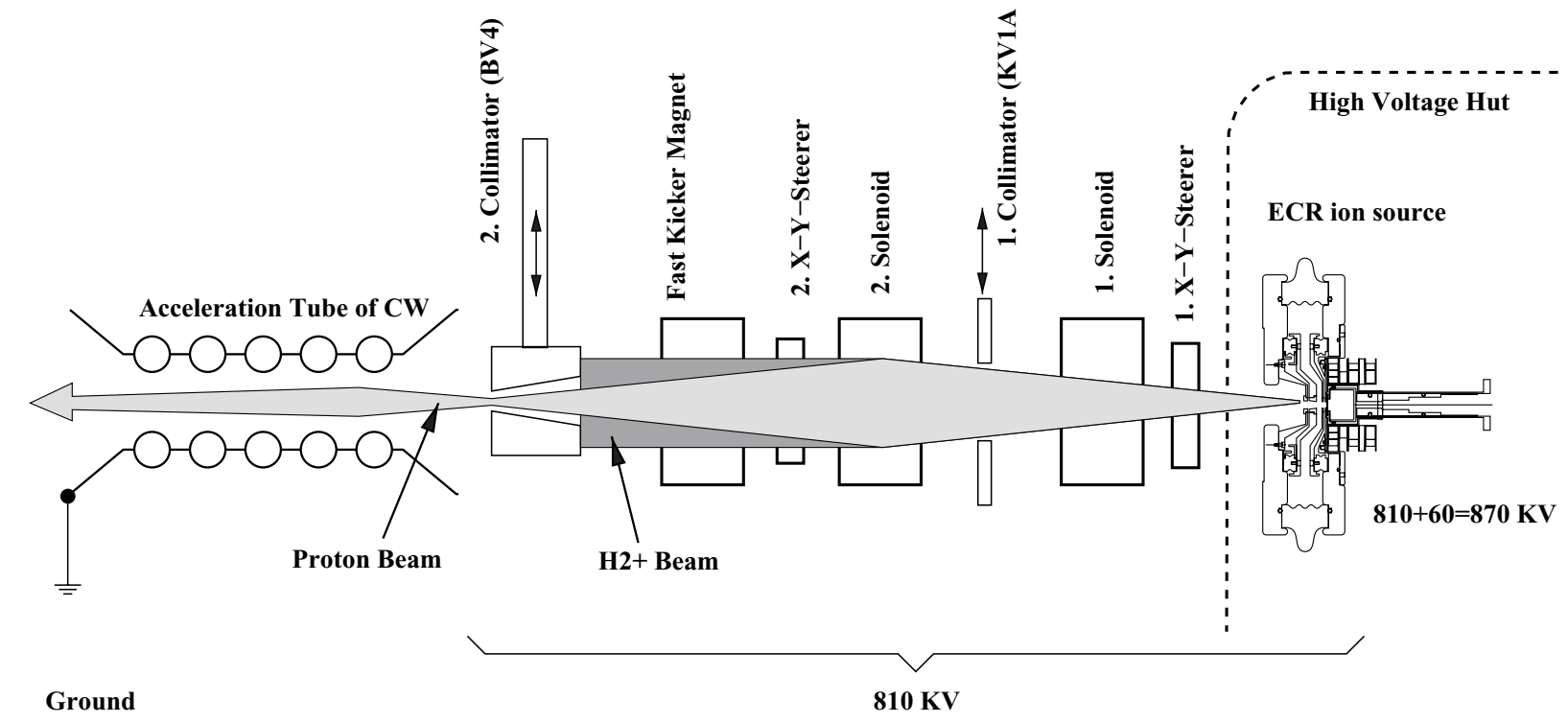

FIG. 10. Low energy beam transport system (LEBT) on the $810 \mathrm{KV}$ high voltage platform of the CW. The protons are focused by two solenoid magnets onto the collimator BV4 (7 mm aperture). BV4 blocks the majority of the $\mathrm{H}_{2}^{+}$-ions which are not focused due to their higher magnetic rigidity compared to protons. Two pairs of steerer magnets enable to adjust beam position and slope. The first collimator KV1A with $35 \mathrm{~mm}$ aperture is used to cut off the tails of the phase space distribution. 

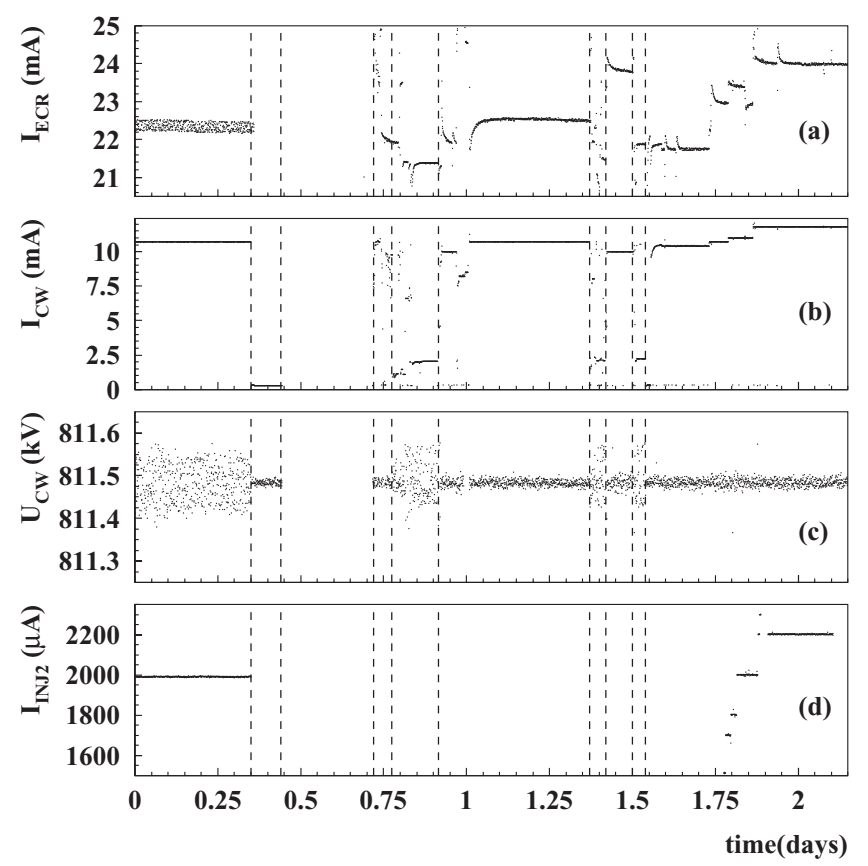

FIG. 11. Comparison of source performance with ac and dc magnetron filament currents. Total extracted ion current (a), current in CW (b), voltage of $\mathrm{CW}$ (c), beam current in INJECTOR II (d). The dashed lines indicate from left to right: Switching ECR off, CW voltage off, beam current in CW started again, and finally (three times): Current reduction by pulsing the kicker magnet in the LEBT with $500 \mathrm{~Hz}$, kicker magnet off again. The $50 \mathrm{~Hz}$-ripple $(0<t<0.35)$ disappeared nearly completely and with the new magnetron assembly the $\mathrm{CW}$-voltage ripple does not increase significantly with beam on $(t>1.6)$ compared to beam off $(0.35<t<0.44)$.

leads to a beam position instability after the first dipole magnet. This jitter causes indeed an increase of the effective beam width and, therefore, an increase of the losses at the extraction elements of the accelerator. The advantage of a dc filament heater current was demonstrated at the ISTS by a test with a set of batteries connected to the filament. A magnetron power supply with dc heating current was ready for installation beginning of July, 2010. Figure 11 compares some parameters before and after installation of the dc magnetron power supply.

The new source was installed mid February, 2010 and is in routine operation since beginning of March. After about $2000 \mathrm{~h}$ of operation, the plasma chamber was inspected during a regular service day on May 25, 2010, though nothing indicated that a service was necessary. The plasma chamber was cleaned by sandblasting the inner walls and then reinstalled. The source failed to restart at once. Normally the plasma ignites at about $20-50 \mathrm{~W}$ of MW power. However, occasionally ignition problems appeared with new or sandblasted plasma chambers. One method to overcome this problem is to use the water leak as the main gas injector, i.e., by igniting a water plasma and subsequently reducing the water vapor supply and increase of the hydrogen flow. This is a cumbersome procedure in the accelerator environment, but easily done at the ISTS. Therefore, we decided to only install chambers that have seen plasma for some hours at the ISTS beforehand. There were no ignition problems using "experienced" chambers since then.
The second plasma chamber was in operation until end of August, i.e., for about $2200 \mathrm{~h}$. It was exchanged since the performance of the source degraded with the time of operation. For the same proton current, ever increasing microwave power had to be applied. Since continuous operation above $700 \mathrm{~W}$ has not been tested for longer periods at the ISTS, we decided not to exceed this limit at the accelerator. Also, vacuum pressure indicated that a (small) leak had developed. Inspection of the plasma chamber showed a spot of about $5 \mathrm{~mm}$ diameter at the chamber bottom covered by a thin silvercolored metal film. The film was thick enough to be electrically conducting. We believe that the air contamination of the gas had increased the sputtering yield. The increased amount of metal ions deposited at the centre of the chamber bottom and formed a short-circuit for the microwave. This incident shows that leak-tightness is essential for long term operation of the source. Tests with a modified design using a metal seal between plasma chamber and base plate are in preparation.

As the up-to-now experience with maintenance shows that the exchange of the plasma chamber requires $2-3 \mathrm{~h}$ only (including pump down time) the risk of an unscheduled interruption of the beam production becomes acceptable. Therefore, the next running periods will be as long as possible in order to collect more information on the long term behaviour of the source.

\section{CONCLUSIONS}

A compact ECR proton source has been developed and installed at the PSI high intensity proton accelerator. The source has an rms-emittance of about $4 \pi \mathrm{mm}$ mrad (normalized $0.045 \pi \mathrm{mm} \mathrm{mrad}$ ) and delivers $10-12 \mathrm{~mA}$ of net proton current with a proton fraction of $70 \%-90 \%$. A water leak provides a small admixture of $\mathrm{H}_{2} \mathrm{O}$ vapor to support a high proton fraction. The beam formation in the extraction system was modeled and the phase space distribution could be reproduced. In order to achieve lowest $50 \mathrm{~Hz}$-ripple the magnetron filament heater was equipped with a dc power supply.

The source was commissioned successfully in the winter shutdown 2009/2010. It turned out to be very reliable and $24 \mathrm{~h}$ continuous operation for three months $(>2000 \mathrm{~h})$ has been repeatedly demonstrated.

\section{ACKNOWLEDGMENTS}

We would like to thank the PSI workshop staff and all involved PSI groups for their support. Our special thank goes to M. Leupi, R. Baumgartner, and G. Bär for their engagement and collaboration.

\footnotetext{
${ }^{1}$ M. Seidel and P. A. Schmelzbach, in Proceedings of the 18th International Conference on Cyclotrons and their Applications, Giardini Naxos, Messina, Italy, 1-5 October 2007, edited by D. Rifuggiato and L. A. C. Piazza (INFN-LNS, Catania, Italy, 2008), pp. 157-162.

${ }^{2}$ M. Seidel, in Proceedings of Hadron Beam, Oak Ridge Nat. Lab., Nashville, TN, USA, 25-29 August 2008. http://accelconf.web. cern.ch/accelconf/

${ }^{3}$ M. Olivo, E. Mariani, and Joseph Sherman, Rev. Sci. Instrum. 63(4), 2714 (1992).
} 
${ }^{4}$ C. Markovits, in Proceedings of the 12th IEEE Particle Accelerator Conference, (PAC), Washington, DC, 16-19 Mar 1987, p. 1954.

${ }^{5}$ M. Olivo, W. Joho, and U. Schryber, IEEE Trans. Nucl. Sci. 26(3), 3980 (1979).

${ }^{6} \mathrm{M}$. Olivo, in Proceedings of the 1984 Linear Accelerator Conference, (LINAC 84), 7-11 May 1984, edited by Norbert Angert, Darmstadt, Germany, 1984, (GSI 84-11), pp. 380-383.

${ }^{7}$ A. J. T. Holmes, E. Thompson, and F. Watters, J. Phys. E.: Sci. Instrum. 14, 856 (1981).

${ }^{8}$ J. Sherman, A. Arvin, L. Hansborough, D. Hodgkins, E. Meyer, J. D. Schneider, H. V. Smith, Jr., M. Stettler, R. R. Stevens, Jr., M. Thuot, and T. Zaugg, Rev. Sci. Instrum. 69, 1003 (1998).

${ }^{9}$ G. Ciavola, L. Celona, S. Gammino, and F. Chines, Rev. Sci. Instrum. 73(2), 1030 (2002).

${ }^{10}$ R. Gobin, P.-Y. Beauvais, D. Bogard, G. Charruau, O. Delferriere, D. De Menezes, A. France, R. Ferdinand, Y. Gauthier, F. Harrault, P. Mattei, K. Benmeziane, P. Leherissier, J.-Y. Paquet, P. Ausset, S. Bousson, D. Gardes, A. Olivier, L. Celona, and J. Sherman, Rev. Sci. Instrum. 75, 1414 (2004).

${ }^{11}$ B. Cui, Y. Ma, L. Li, C. Jiang, and W. Jiang, Rev. Sci. Instrum. 75, 1457 (2004).

${ }^{12} \mathrm{Sm}_{2} \mathrm{Co}_{17}$-Magnets Vacomax from Vakuumschmelze.

${ }^{13}$ P. A. Schmelzbach, A. Barchetti, H. Einenkel, and D. Goetz, in Proceedings of 18th International Conference on Cyclotrons and their Applications, Giardini Naxos, Italy, 1-5 October 2007, edited by D. Rifuggiato and L. A. C. Piazza (INFN-LNS, Catania, Italy, 2008), pp. 292-294.
${ }^{14}$ Kalrez 4079, operating temperatures up to $315^{\circ} \mathrm{C}$.

${ }^{15}$ T. Taylor and J. F. Mouris, Nucl. Instrum. Methods Phys. Res. A 336, 1 (1993).

${ }^{16}$ Sairem Microwave generator GMP 12 KSM (2.45 GHz, $\left.1200 \mathrm{~W}\right)$, magnetron YJ 1540-3 (watercooled) with circulator and waterload, specially equipped with dc-filament heater current.

${ }^{17}$ Sairem Automatic 4-stub tuner AI 4 SA-2450/340 EL 1097.

${ }^{18}$ L. Celona, G. Ciavola, and S. Gammino, Rev. Sci. Instrum. 69(2), 1113 (1998).

${ }^{19}$ C. Baumgarten, Contr. to European Cyclotron Progress Meeting ECPM XXXVI, Hahn-Meitner-Institut, Berlin, Germany, 15-18 October 2008 (unpublished); European Cyclotron Progress Meeting ECPM XXXVII, KVI Groningen, Netherlands, 28-31 October 2009 (unpublished).

${ }^{20}$ Vladimir P. Derenchuk, Rev. Sci. Instrum. 75(5), 1851 (2004).

${ }^{21}$ see for instance Z. Q. Xie in Proceedings of the 1997 IEEE Particle Accelerator Conference p. 2662-2666 and references therein.

${ }^{22} \mathrm{D}$. Spence and Keith R. Lykke, in IEEE Proceedings of the 1995 Particle Accelerator Conference, Dallas, Texas, 1-5 May 1995 (IEEE, Piscataway, NJ, 1996), 5v. (IEEE Catalog No. 95CH35843), pp. 1019-1021; D. Spence, G. McMichael, K. R. Lykke, J. D. Schneider, J. Sherman, R. Stevens, and D. Hodgkins, Rev. Sci. Instrum. 67(4), 1642 (1996).

${ }^{23}$ S. Zou, L. Pichl, M. Kimura, and T. Kato, Phys. Rev. A 66, 042707 (2002).

${ }^{24}$ RELAX3D User's Guide, see http://legacyweb.triumf.ca/compserv/relax3d. html. 